\title{
Time-dependent characterisation of stability performance of EJ-309 detector systems
}

\author{
Vytautas Astromskas ${ }^{1, *}$ and Malcolm Joyce ${ }^{1, *}$ \\ ${ }^{1}$ Engineering Department, Lancaster University, Bailrigg, Lancaster, LA1 4YW, United Kingdom
}

\section{INTRODUCTION}

Multi-element scintillator systems are increasingly used in a broad range of applications such as neutron spectrometry [1], nuclear security [2], nuclear reaction [3] positron emission tomography [4] and all detector are required to operate as identical to each other as possible. However, typically, there is a significant difference in the performance of the scintillator material, the photomultiplier tubes and/or the quality of the coupling which leads to variance in the detectors performances. This is especially important in material discrimination applications in mixed radiation fields, where a non-linear or non-matching linearity with regards to energy response can lead to false identification or discrimination of the material. Normally, the detectors are calibrated using laboratory-based radioactive gamma sources to account for the difference in energy linearity. However, this method ignores the differences in gain which is important for multiplicity assays. Finally, the knowledge of stability of the system is vital for real-world applications. The focus of this study is to investigate the normalisation of the performance of a multi-element scintillator detector system as well as the stability with respect to time and temperature.

\section{Methods}

Seven EJ-309 liquid scintillators coupled to photomultiplier tube (PMT) were tested in terms of the uniformity of their response to gamma radiation sources. The scintillator detectors were obtained from Scionix, Netherlands. The liquid scintillator was housed in a $3 \mathrm{~mm}$ thick aluminium chamber with the dimensions of 10x10x10 cm. The PMTs were connected to a Hybrid Instruments single-channel mixed field analyzer (MFA) digitizer system for signal processing and data readout. Laboratory radioactive sources, namely, Cesium-137, Sodium22 and Cobalt-60 were used to evaluate the uniformity of the detectors' response in terms of energy linearity and efficiency.

For all experiments, a radioactive source was placed at the centre on top of the vertically held detector. For energy response and efficiency measurements, the spectrum was collected for 10 minutes in the case of Cs-137, and for 30 minutes for Na-22 and Co-60 due to different activity levels of the sources. For stability measurement, the spectrum was continuously collected for 3 hours using Cs-137 radioactive

${ }^{*}$ V.Astromskas and M.Joyce
rigg, Lancaster, LA1 4 YW, U.K (e-maith Lancaster University, Bail-
artromskas @lancaster.ac.uk; m.joyce@lancaster.ac.uk) source. The detectors were energy calibrated by adjusting the applied high voltage in such way that the Cs-137 peak would be centred at the channel 200 on the MFA software. The same applied HV was, then, kept for Na-22 and Co-60 spectra in order to identify the deviations in energy linearity. The applied high voltages ranged from $-1576 \mathrm{~V}$ to $-1705 \mathrm{~V}$.

\section{RESUlts AND Discussion}

The energy response of all seven detectors is shown in figure 1. The peak positions were extracted from the Gaussian fit of each individual peak. The extent of the error bars is contained within the data point. The figure indicates that the detectors have a non-uniform response with respect to each other as well as that the greater non-uniformity is observed in the high energy channels. This could be due to non-linear response in terms of energy of the PMTs or due to the higher energy peak's channel being further away from the calibration channel. The effect will be investigated and identified in future work.

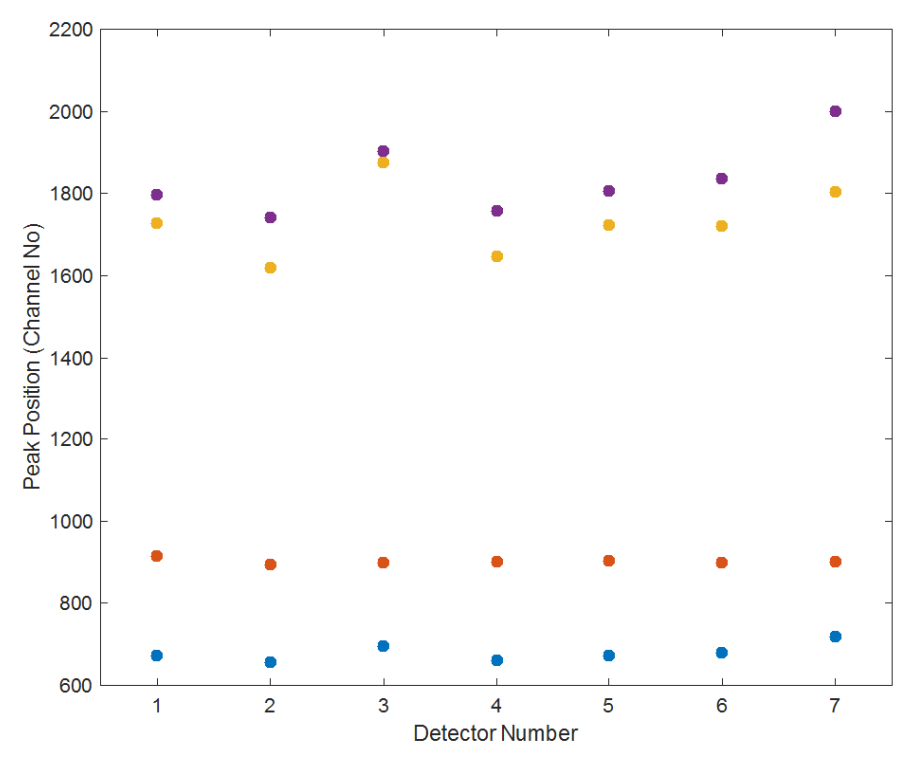

Fig. 1. The response in terms of energy for each of the seven detectors..

The linearity of the energy response is shown in figure 2 . The peak positions and the error bars were extracted from the Gaussian fit. The extracted R-Square values extracted from the linear fit ranged from 0.871 to 0.927 , thus, further supporting the further investigation in the energy response of the detectors is required in order to ensure uniform response in multidetector arrangements. 


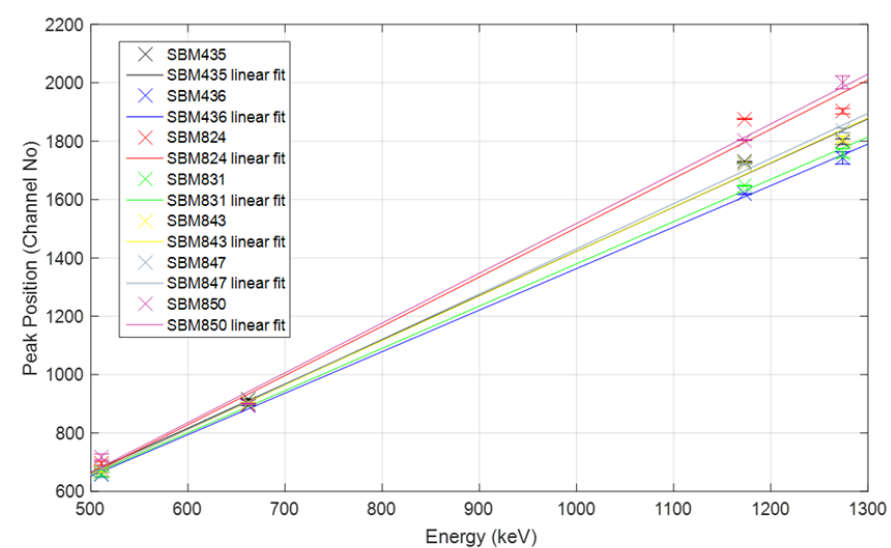

Fig. 2. The energy linearity of each of the detectors. The peak positions were fitted with linear fit.

Furthermore, the efficiency of the detectors was investigated and the results are displayed in figure 3 . The errors bars are a combination of the statistical error of the total number of counts collected and the uncertainty in the fit. The greater error bars appear fig.3.(c) and fig.3.(d) due to lower activities of the sources. Despite all detectors being calibrated using the Cs-137 source, the difference in efficiencies is observed in fig.3.(b) as well. The efficiencies varied by up to $24 \%$. Interestingly, the significant difference in efficiencies was observed in detectors 1 and 6 despite the fact both of these detectors had applied high voltage set to $-1700 \mathrm{~V}$. This indicates that the difference in efficiency is not caused by applied high voltage.

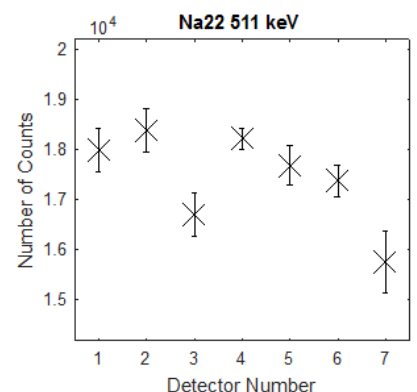

(a)

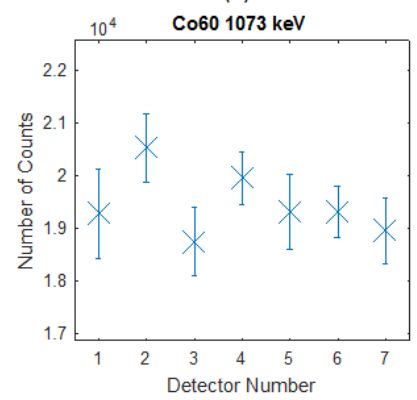

(c)

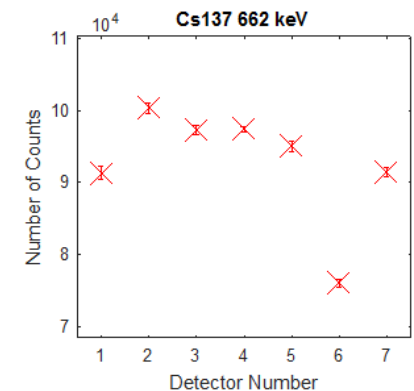

(b)

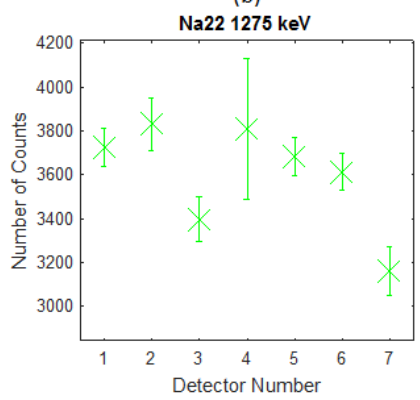

(d)
Fig. 3. The efficiency of the seven detectors for: (a) Na-22 $511 \mathrm{keV}$ peak; (b) Cs-137 $662 \mathrm{keV}$ peak; (c) Co-60 $1073 \mathrm{keV}$ peak; (d) Na-22 $1275 \mathrm{keV}$ peak.

Finally, the stability of a detector over 3 hour period is shown in figure 4. The data shown is incremented in 10 minute steps. The error bars were extracted from the statistical error of the total number of counts collected. The raw data was smoothed using a 10-point filter in order to qualitatively evaluate the stability of the detector. The figure indicates two behaviours. Firstly, that the detector requires a warm-up time of approximately 60 minutes in order to achieve a stable response. Secondly, the detector's operation is stable for the remainder of the experiment. In the future, the stability will be evaluated of all the detectors as well as the dependency of the warm-up time in terms of temperature as this is vital information for applying these EJ-309 liquid scintillator detectors in real-world applications.

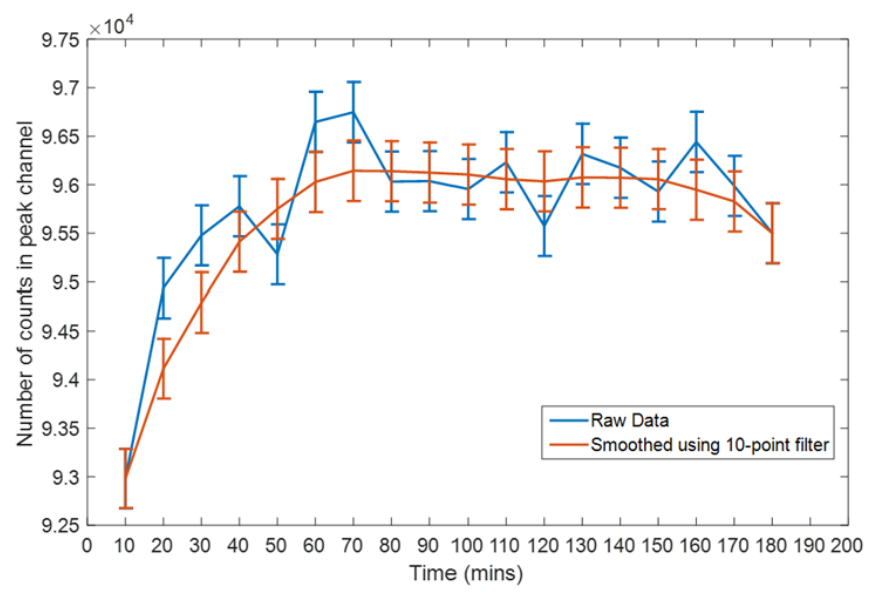

Fig. 4. The number of counts collected over 3 hour period using the Cs-137 radioactive source.

\section{SUMMARY}

In this paper, the stabilization in terms of energy response, time and temperature of multiple EJ-309 liquid scintillator detectors coupled to PMTs is going to be discussed. The results will include a detailed study of the energy response, warm-up time, as well as, temperature effect on the stability of multiple detectors. The final outcome of this research will suggest a methodology for stabilizing a multi-element scintillator system in terms of the aforementioned factors.

\section{REFERENCES}

[1] R. Machrafi, A. L. Miller, and N. Khan, "New approach to neutron spectrometry with multi element scintillator," Radiation Measurements, vol. 80, pp. 10-16, Sept. 2015.

[2] G. Popescu, S. Herman, S. Glover, and H. Spitz, "Compton background suppression with a multi-element scintillation detector using high speed data acquisition and digital signal processing," Journal of Radioanalytical and Nuclear Chemistry, vol. 307, pp. 1949-1955, Mar. 2016.

[3] M. Febbraro, F. D. Becchetti, R. O. Torres-Isea, M. Ojaruega, A. M. Howard, J. J. Kolata, A. Roberts, and A. N. Villano, "Neutron Spectroscopy Without Time-of-Flight Measurement: A DSP-Based Deuterated Scintillator Array," IEEE Transactions on Nuclear Science, vol. 60, pp. 890-896, Apr. 2013.

[4] J. Y. Yeom, R. Vinke, V. C. Spanoudaki, K. J. Hong, and C. S. Levin, "Readout Electronics and Data Acquisition of a Positron Emission Tomography Time-of-Flight Detector Module With Waveform Digitizer," IEEE Transactions on Nuclear Science, vol. 60 , pp. 3735-3741, Oct. 2013. 